\title{
Quaderni
}

QUADERNI Communication, technologies, pouvoir

\section{Christophe Roux, Corse française et Sardaigne italienne. Fragments périphériques de construction nationale.}

\section{Aurélien Lignereux}

\section{(2) OpenEdition \\ Journals \\ Édition électronique \\ URL : http://journals.openedition.org/quaderni/1089 \\ DOI : 10.4000/quaderni.1089 \\ ISSN : 2105-2956 \\ Éditeur \\ Les éditions de la Maison des sciences de l'Homme}

Édition imprimée

Date de publication : 5 mai 2017

Pagination : 111-114

Référence électronique

Aurélien Lignereux, "Christophe Roux, Corse française et Sardaigne italienne. Fragments

périphériques de construction nationale. », Quaderni [En ligne], 93 | Printemps 2017, mis en ligne le 05 mai 2017, consulté le 24 septembre 2020. URL : http://journals.openedition.org/quaderni/1089 ; DOI : https://doi.org/10.4000/quaderni.1089 


\section{Compte rendu}

Corse française et Sardaigne italienne. Fragments périphériques de construction nationale. Christophe Roux

L'Harmattan, coll. "Logiques politiques", Paris, 2014

par Aurélien Lignereux

Sciences Po Grenoble

1. O. Roynette, Bons pour le service. L'expérience de la caserne en France à la fin du XIXe siècle, Paris, Belin, 2000.
La Corse est devenue française en 1768 et la Sardaigne piémontaise depuis 1718 puis italienne à partir de 1861 ; reste à savoir comment on a fait des Corses des Français, et des Sardes des Italiens. C'est à cette problématique dérivée du mot d'Azeglio qu'entend répondre Christophe Roux. Il s'agit de saisir sur le vif le processus de construction nationale, dans une visée doublement comparative (France-Italie, Corse-Sardaigne), en passant au crible trois grands leviers de nationalisation : la scolarisation, les migrations et la politisation. On peut regretter que la valeur intégrative du service militaire ait été minorée au prétexte que l'expérimentation du territoire national s'avère limitée, comme si le rôle de l'armée se bornait à dépayser les hommes au fil des garnisons, alors que l'acculturation militaire passe d'abord par l'espace propre qu'elle produit, celui de la caserne et de ses normes ${ }^{1}$. Il n'empêche que le dispositif de recherche annoncé est tout à fait séduisant pour qui veut percer la fabrique de l'État-Nation à l'épreuve de ses périphéries : a priori, rien n'illustre mieux pareil façonnement que le sort contrasté de ces « îles sœurs ». En réaction à l'écheveau de modèles élaborés par toute une lignée de travaux de science politique, que C. Roux passe en revue de façon expéditive (jusqu'à douze références en vingt lignes, p. 14), la thèse mise sur les vertus d'une observation empirique des interactions entre sociétés locales et desseins étatiques. En définitive, le pari est double : défiger les réputations acquises ; démontrer l'intérêt revigorant d'une collecte de première main. Or il n'est qu'à moitié tenu.

Le parallèle France-Italie, opéré méthodiquement entre des législations convergentes et des institutions jumelles, écorne l'idée établie qui érige la 
France en modèle et l'Italie en mauvais élève, car cette dernière peut ouvrir la voie sur le plan des principes, à l'exemple de la gratuité scolaire (lois Casati et Coppino des 13 novembre 1859 et 15 juillet 1877). La rhétorique de l'intégration à la française se heurte, quant à elle, à l'écueil corse du fait d'une emprise clientéliste que relancent la captation des mandats républicains et l'extension du périmètre de l'action publique. Le premier chapitre valide la légitimité d'une comparaison entre les deux îles : la mise en perspective est sans doute cavalière (on ne trouve pas trace de l'opposition, si sensible aux yeux des hommes du $18^{\mathrm{e}}$ siècle, entre les insulaires du littoral et ceux de l'intérieur), mais elle suffit à dégager un même passé de tutelle au moindre coût exercée tant par la république de Gênes que par la couronne d'Aragon. La similitude se retrouve dans les phases révolutionnaires, autour de Paoli et d'Angioy, du fait de l'esprit notabiliaire qui anime les Requêtes générales et les Cinq demandes. Cet accommodement avec ce qui existe serait une formule promise à durer puisque $\mathrm{C}$. Roux ne craint pas de conclure que la Troisième République et la monarchie italienne devront s'en satisfaire.

En revanche, la défense et illustration d'une recherche empirique laisse insatisfait faute d'une mise en pratique convaincante. Les deux tiers du $\mathrm{XIX}^{\mathrm{e}}$ siècle sont survolés et réduits au rang de prélude aux années 1870-1914. N'est-ce pas s'aligner sur une vulgate vieillie quand on sait, par exemple, le poids que l'historiographie accorde désormais plus volontiers au Second Empire ? La lacune est d'autant plus criante que le cent-cinquantenaire de 1860 a renouvelé les approches tant de la réunion de Nice et de la Savoie que du Risorgimento, dans une perspective à la fois européenne et attentive au jeu des familles ou des réseaux. Ces apports auraient été précieux pour le présent ouvrage tiré d'un doctorat en science politique soutenu fin 2005, et dès lors à contretemps des questionnements actuels : la seule comparaison bilatérale ne saurait rendre compte de rythmes qui, à l'ère démocratique et médiatique, sont transnationaux.

N'est-ce pas aussi l'un des effets pervers de l'affiliation à Eugen Weber ? Le chapitre II consiste en une apologie de La Fin des terroirs, afin d'en révéler le soubassement théorique sous ses dehors impressionnistes, et d'en réfuter les principales critiques - tout en en admettant la méconnaissance fâcheuse du cumul possible des identités. Cette inspiration quasi exclusive a isolé C. Roux des dynamiques historiennes actuelles, l'amenant à déplorer le déficit d'études de la construction nationale dans ses aspects concrets. $\mathrm{Ne}$ 
2. P. M. Jones, Politics and rural society. The southern Massif Central c. 1750-1880, Cambridge, CUP, 1985.

3. J.-N. Luc, La Statistique de l'enseignement primaire, $X I X^{e}-X X^{e}$ siècles : politique et mode d'emploi, Paris, EconomicaINRP, 1985.

4. P. Karila-Cohen, "Les préfets ne sont pas des collègues. Retour sur une enquête ", in Genèses, 2010-2, $n^{\circ} 79$, pp. 116-134. suffisait-il pas de se tourner vers l'essor de l'histoire des polices pour plonger via les gendarmes et les carabiniers dans le quotidien en mue des populations? L'attention portée aux pratiques judiciaires, aux usages qu'en font les justiciables, offre ces données de terrain que l'auteur juge inexistantes. C'est d'autant plus vrai que, point commun avec E. Weber, cette historiographie a privilégié les espaces périphériques. On regrettera donc que la comparaison n'ait pas été transrégionale. La Sardaigne n'est-elle pas une pièce du Mezzogiorno ? Le constat qu'opérait Peter Jones pour le sud-est du Massif central de l'acceptation d'un cow-milk State est sans doute transposable à la Corse $^{2}$; la « composition française » se laisse percevoir dans le chevauchement des identités qu'étudie Mona Ozouf à l'échelle fine de son enfance bretonne. Les travaux relevant de «l'école pyrénéenne » sont absents, alors qu'ils apportent non seulement un exemple de trajectoire de la dissidence à l'intégration mais surtout un modèle pour surmonter l'obstacle des sources. De fait, c'est une démarche compréhensive qu'il fallait mener; ce sont des bribes de parole insulaire qu'on attendait car tels sont les « fragments » probants.

En lieu et place, l'enquête puise pour l'essentiel à deux filons discutables. D’une part, les statistiques utilisées sont exclusivement tirées de séries d'époque : le raisonnement se fonde dès lors sur des indicateurs dont on ignore les filières de constitution. Les déchiffrer exige un mode d'emploi, tel celui livré naguère par J.-N. Luc (1985)³. Voilà qui aurait évité de succomber aux illusions (les « chiffres sont ici particulièrement éloquents », p. 89), ou d'avoir à composer avec des décomptes friables comme en matière d'absentéisme, de démographie ou de corps électoral («il semble vain d'essayer d'analyser plus dans le détail des chiffres dont on connaît la fragilité », p. 190). Si l'on peut s'y résigner malgré tout, il n'en va pas de même pour la place prise par les citations. C. Roux laisse les contemporains, tous représentants de la société englobante, gloser sur ces chiffres. C'est oublier que «les préfets ne sont pas des collègues ${ }^{4} »$ : leurs analyses ne font autorité que dans le cadre du paradigme d'État dont ils sont les porte-parole. Sans doute, à l'occasion, les propos d'un vice-recteur ou d'un journaliste sont-ils nuancés (« Ce compterendu n'est pas exempt d'exagération mais il se fait l'écho » p. 114). Or le problème ne relève pas d'une question de degré, mais tient à la nature de discours qu'il reste à déconstruire pour exhiber leur système de représentations, car peut-on citer (p. 138) ce que dit le consul italien à Bastia sur les «qualités guère fréquentes chez le Corse » (docile, actif, sobre), sans rappeler, entre autres, les poncifs hérités de l'Antiquité ? Le mieux n'aurait-il pas été 
de changer d'échelle ? C'est en descendant au niveau d'un canton que pouvait être dévoilé le jeu des acteurs, au révélateur de dossiers circonscrits tels que l'assistance publique. Bref, on peut déplorer le manque de proximité avec le terrain, impression que conforte l'absence de cartes.

Position inconfortable du chercheur assez courageux pour naviguer entre les lignes, entre le schématisme modélisateur des politistes et le pointillisme sourcilleux des historiens ! Ces remarques sont en effet à lire comme des regrets à la hauteur de l'intérêt suscité par cette recherche novatrice, et font d'ailleurs écho au constat final de l'auteur, qui souligne l'écueil des sources et reconnaît que « la tension incontournable entre travail intensif et extensif a penché dans ce livre du côté de la seconde branche de l'alternative» (p. 202). Il mise sur le caractère cumulatif des travaux à venir pour approfondir une sociologie historique de l'État-nation dont il pose les bases. Car, en l'état, l'enquête est déjà riche d'acquis : parallèle vivifiant entre la France et l'Italie, comparaison féconde entre la Corse et la Sardaigne, elle aboutit à constater une insertion nationale plus avancée de la première mais qui ne suffit pas à venir à bout du clientélisme. 